NASA Technical Memorandum 105406

AIAA-92-0145

\title{
Pressure Wave Propagation Studies for Oscillating Cascades
}

Dennis L. Huff

Lewis Research Center

Cleveland, Ohio

Prepared for the

30th Aerospace Sciences Meeting and Exhibit

sponsored by the American Institute of Aeronautics and Astronautics

Reno, Nevada, January 6-9, 1992 


\title{
PRESSURE WAVE PROPAGATION STUDIES FOR OSCILLATING CASCADES
}

\author{
Dennis L. Huff \\ NASA Lewis Research Center \\ Cleveland, Ohio
}

\begin{abstract}
The unsteady flow field around an oscillating cascade of flat plates is investigated using a time-marching Euler code. Exact solutions based on linear theory serve as model problems to study pressure wave propagation in the numerical solution. The importance of using proper unsteady boundary conditions, grid resolution and time step size is demonstrated. Results show that an approximate non-reflecting boundary condition based on linear theory does a good job of minimizing reflections from the inflow and outflow boundaries and allows the placement of the boundaries to be closer than cases using reflective boundary conditions. Stretching the boundary to dampen the unsteady waves is another way to minimize reflections. Grid clustering near the plates does a better job of capturing the unsteady flow field than cases using uniform grids as long as the CFL number is less than one for a sufficient portion of the grid. Results for various stagger angles and oscillation frequencies show good overall agreement with linear theory as long as the grid is properly resolved.
\end{abstract}

\section{Nomenclature}

$\begin{array}{ll}\bar{A} & \begin{array}{l}\text { Roe matrix } \\ \text { chordlength, speed of sound or } \\ \text { unsteady characteristic variables } \\ \text { pressure difference }\end{array} \\ \triangle \mathrm{CP} & \begin{array}{l}\text { coefficient, } \\ \left(p_{-}-p_{.}\right) /\left(\rho_{1} V_{1}^{2}\left|h_{1}\right| k\right)\end{array} \\ \mathrm{e} & \begin{array}{l}\text { energy } \\ \text { flux vectors }\end{array}\end{array}$

Copyright c 1992 by the American Institute of Aeronautics and Astronautics, Inc. No copyright is asserted in the United States under Title 17, U.S. Code. The U.S. Govemment has a royalty-free license to exercise all rights under the copyright claimed herein for Governmental purposes. All other rights are reserved by the copyright owner.

\begin{tabular}{|c|c|}
\hline \multirow[t]{2}{*}{$h_{1}$} & amplitude of oscillation for \\
\hline & $\begin{array}{l}\text { plunging motions based on } \\
\text { chordlength }\end{array}$ \\
\hline $\operatorname{Im}\{\}$ & imaginary part of \{\} \\
\hline k & $\begin{array}{l}\text { semi-chord reduced } \\
\text { frequency, } \omega c /\left(2 V_{1}^{2}\right)\end{array}$ \\
\hline M & Mach number \\
\hline $\mathbf{p}$ & static pressure \\
\hline Q & dependent variable vector \\
\hline $\operatorname{Re}\{\}$ & real part of \{\} \\
\hline$t, \tau$ & time \\
\hline$u, v$ & $\begin{array}{l}\text { velocities in the } \mathrm{x}, \mathrm{y}- \\
\text { directions, respectively }\end{array}$ \\
\hline V & total velocity \\
\hline $\mathrm{x}, \mathrm{y}$ & spatial coordinates \\
\hline Y & stagger angle, defined as angle \\
\hline & $\begin{array}{l}\text { between locus of leading edge } \\
\text { points and y-axis }\end{array}$ \\
\hline$\xi . \eta$ & curvilinear coordinate directions \\
\hline$\rho$ & fluid density \\
\hline$\sigma$ & interblade phase angle \\
\hline$\omega$ & oscillation frequency \\
\hline
\end{tabular}

Subscripts

1,2 conditions at inlet/exit

,$+-\quad$ upper/lower surfaces on plate

$L, R \quad$ left/right of an interface

\section{Introduction}

The accurate prediction of the unsteady flow field in turbomachinery remains a critical challenge for predicting noise and aeroelastic response. Advanced designs of ducted propellers for large commercial 
aircraft have relatively large shroud diameters with swept composite blades. The blades are expected to be more flexible than the blades used in current turbofans. Blade row interaction effects will be important for both aeroelasticity and aeroacoustics. Analytical and experimental predictions of the unsteady aerodynamics associated with these designs are needed to ensure safe operation and lower noise.

While three-dimensional methods are needed for a complete simulation of the unsteady flows associated with swept blades, two-dimensional analyses of linear cascades are used in practice. Aeroelastic and aeroacoustic analyses commonly model a single blade row responding to a gust or blade vibration. There are a number of numerical and analytical methods available for addressing the two-dimensional, oscillating cascade problem. Perturbation methods have been used the most since they do a reasonably good job near design conditions and are inexpensive to compute. Also, exact specification of the inflow and outflow boundary conditions is possible. An assumption made with all of these schemes is that the unsteady flow field is a small perturbation of the mean flow, which allows the unsteady flow to be linearized. Classical linearization about a uniform mean flow has been done by Smith (ref.1) and Whitehead (ref.2). Verdon (ref.3), Hall/Crawley (ref.4) and Fang/Atassi (ref.5), who account for perturbations about a non-uniform meanflow. Time-marching, non-linear schemes are also available that solve the full-potential (ref.6), Euler (refs.7-12), and Navier-Stokes (refs.8,11) equations. These methods are necded for flows where small-perturbation approaches are no longer valid. However, these methods require larger amounts of computer time compared to linearized methods. In addition, proper treatment of the inflow and outflow boundary conditions is difficult, but essential for accuracy.

The objective of this paper is to investigate the unsteady flow field around an oscillating cascade using a time-marching Euler code. The code solves the non-linear Euler equations using a high resolution wave-split scheme. Only linear solutions are sought for a simple cascade of flat plates. Using an Euler code is overkill for this problem, but an exact solution is known from linear theory that can be used as a baseline for code validation. Furthermore, fundamental studies of pressure wave propagation using a time-marching CFD code are necded to better understand the limitations of numerical solutions. Choosing the proper grid resolution, time step size and boundary conditions are important for resolving the unsteady flow field. Modeling the linear flow for oscillating flat plates is a first step toward validating a numerical solution used for acroelasticity and acroacoustics, and needs to be modeled correctly before solutions for more complex flow fields can be considered relevant. This is particularly true for moderate to high blade passing frequencies associated with forced response problems in aeroelasticity and blade row interaction problems in aeroacoustics.

In the present work, the numerical predictions of the unsteady aerodynamics for an oscillating cascade of flat plates is studied in detail. Oscillation frequencies are chosen that are representative of blade passing frequencies for an advanced ducted propeller design. The importance of using the proper inflow and outflow boundary conditions is demonstrated. The effects of the boundary distance from the cascade, grid distribution and time step size on the solution are investigated.

\section{Governing Equations and Numerical Algorithm}

The unsteady two-dimensional Euler equations written in conservative differential form are used and are transformed from a Cartesian to a time-dependent curvilinear reference frame. This transformation process and complete details of the numerical algorithm are presented in references 13-17. A brief overview is given below.

The transformed equations can be written in vector form as

$$
\frac{\partial Q}{\partial \tau}+\frac{\partial F}{\partial \xi}+\frac{\partial G}{\partial \eta}=0
$$

where

$$
\begin{gathered}
Q=J q \\
F=J\left(\xi_{1} q+\xi_{x} f+\xi_{y} g\right) \\
G=J\left(\eta_{1} q+\eta_{x} f+\eta_{y} g\right)
\end{gathered}
$$


and

$$
\begin{gathered}
q=[\rho, \rho u, \rho v, e]^{T} \\
f=\left[\rho u, \rho u^{2}+\rho, \rho u v, u(e+p)\right]^{\top} \\
g=\left[\rho v, \rho u v, \rho v^{2}+p, v(e+p)\right]^{T}
\end{gathered}
$$

$\xi_{1}, \xi_{x}, \xi_{y}, \eta_{1}, \eta_{x}$ and $\eta_{y}$ are the metrics and $J$ is the Jacobian of transformation.

These equations are discretized and solved using a finite volume method, where cell centers are denoted as $\mathrm{i}$ and $\mathrm{j}$ :

$$
\frac{\partial Q}{\partial \tau}+\frac{\delta_{i} F}{\Delta \xi}+\frac{\delta_{i} G}{\Delta \eta}=0
$$

With $\Delta \xi=\Delta \eta=1$ (by definition), this becomes

$$
\frac{\partial Q}{\partial \tau}=-(\delta, F+\delta, G)
$$

where

$$
\delta .(.)=(.) \cdot+1 / 2-(.) \cdot-1 / 2 \text {. }
$$

The components of the dependent vector, $Q$, represent average values over a particular cell. However, a method is needed to allow these fluxes to be accurately represented at cell faces. As discussed in reference 13 , the method used in the present effort is based on the one-dimensional approximate Riemann solver of Roe (ref.18) at cell interfaces for each coordinate direction. The method uses an approximate equation to represent a quasilinear form of a locally one-dimensional conservation law:

$$
\frac{\partial q}{\partial t}+\bar{A}\left(q_{L}, q_{R}\right) \frac{\partial q}{\partial x}=0
$$

where $\bar{A}\left(q_{L}, q_{R}\right)$ is a constant matrix representative of local cell interface conditions and is constructed using so-called "Roe averaged" variables. The determination of the eigensystem of $\bar{A}$ and knowing that the change in dependent variables across an interface is proportional to the right eigenvectors allows first order flux formulae to be constructed. This approach of extracting flow field information from characteristically dictated directions is commonly referred to as flux difference splitting (FDS) and is applicable to multidimensional space, so long as the assumption is made that all wave propagation occurs normal to a particular cell interface. To provide higher order spatial accuracy, a corrective flux is appended to the first order flux discussed above. In addition, in order to control dispersive errors commonly encountered with higher order schemes, so-called "limiters" are used to limit components of the interface flux resulting in total variation diminishing (TVD) schemes. All solutions presented herein were obtained using the basic algorithm developed in reference 13 , which is third-order accurate spatially and second-order accurate in time.

\section{Grid}

The flow equations are solved on Cartesian H-grids. Uniformly spaced grids are generated algebraically.

Grids with cells clustered toward the plates or coarsened near the inflow/outflow boundaries were generated using a two-dimensional version of the IGB code developed by Beach (ref.19) for turbomachinery. This grid generator uses a hyperbolic tangent function to determine the spacing upstream and downstream of the cascade and ensures smooth variation in grid spacing. Once a grid is generated for a single passage, it is stacked to form a cascade with multiple passages.

All applications are for plunging motions of plates with an interblade phase angle, $\sigma=90$ degrees. Based on the direct store method presented in reference 12 , this requires four blade passages. The Euler code is blocked such that the passage-centered $\mathrm{H}$-grids and the corresponding dependant variables are stored on a SSD (Solid-State Storage Device) on the CRAY computer and retrieves the information as needed. Within one block, the lower computational boundary contains the upper surface of one blade in the cascade, while the upper computational boundary contains the lower surface corresponding to the adjacent blade. The grid from each passage is deformed such that the grid follows the plunging motion near the plates and the grid near the center of the passage remains fixed. This is done using weighting functions and is described in more detail in reference 12 . The code time-marches the grid and the flow solution for harmonic oscillations of the plates. 


\section{Boundary Conditions}

\section{Solid Surface and Periodic Boundary Conditions}

The solid surface boundary conditions used in these studies implement zero pressure gradient conditions. Other forms of boundary conditions have been investigated by Janus (ref.20) and others at Mississippi State University and found that the zero pressure gradient boundary conditions are sufficient as long as the grid near the surface is adequately resolved. The boundaries that extend upstream and downstream of the plates are called "periodic boundaries" for plates with harmonic motion and a specified interblade phase angle. The Euler code stores phantom cells on either side of these boundaries and uses injection of the dependant variables to specify the boundary conditions for a given passage.

\section{Steady Inflow and Outflow Boundary Conditions}

The far-field, steady boundary conditions are based on characteristic variables and assume a locally one dimensional flow at the boundary. A summary of their derivation is given below based on reference 20 .

For the sake of deriving the boundary conditions, the Euler equations are written in their non-conservative form:

$$
\frac{\partial q}{\partial \tau}+\alpha \frac{\partial q}{\partial \xi}+b \frac{\partial q}{\partial \eta}=0
$$

The matrices $\alpha$ and $b$ are determine through an eigenvalue analysis. Multiplying by $P_{\xi}^{-1}$ and neglecting the derivatives in the $\eta$ direction gives:

$$
P_{\xi}^{-1} \frac{\partial q}{\partial \tau}+P_{\xi}^{-1} P_{\xi} \wedge_{\xi} P_{\xi}^{-1} \frac{\partial q}{\partial \xi}=0
$$

where $\Lambda_{\xi}$ is a diagonal matrix containing the eigenvalues, $\lambda_{\xi}$; and $P_{\xi}$ and $P_{\xi}^{-1}$ are the left and right eigenvectors, respectively. The characteristic vector is defined as:

$$
W_{k}=P_{k}^{-1} q
$$

$P_{\xi}$ is derived in reference 20 such that the elements of the characteristic vector become:

$$
\begin{aligned}
w_{\xi}^{1}= & \frac{J}{|\nabla \xi|}\left[\xi_{x}\left(\rho-\frac{p}{c_{1}^{2}}\right)\right] \\
w_{\xi}^{2}= & \frac{J}{|\nabla \xi|}\left[\xi_{y}\left(\rho-\frac{p}{c_{1}^{2}}\right)\right] \\
w_{\xi}^{3}= & \frac{J}{\sqrt{2}|\nabla \xi|}\left[\frac{p \nabla \xi}{\rho_{1} c_{1}}+\right. \\
& \left.\left(\xi_{x} u+\xi_{y} v\right)\right] \\
w_{\xi}^{4}= & \frac{J}{\sqrt{2}|\nabla \xi|}\left[\frac{p \nabla \xi}{\rho_{1} c_{1}}-\right. \\
& \left.\left(\xi_{x} u+\xi_{y} v\right)\right]
\end{aligned}
$$

The corresponding eigenvalues are:

$$
\begin{gathered}
\lambda_{\xi}^{1} \cdot \lambda_{\xi}^{2}=\xi_{1}+\xi_{x} u+\xi_{y} u \\
\lambda_{\xi}^{3}=\left(\xi_{x} u+\xi_{y} v\right)+c|\nabla \xi| \\
\lambda_{\xi}^{4}=\left(\xi_{x} u+\xi_{y} v\right)-c|\nabla \xi|
\end{gathered}
$$

The implementation of the characteristic variable boundary conditions requires knowing if the boundary is an inflow or an outflow. Generally, this is done by computing the sign of $\lambda$ to determine the directions of the characteristics. However, for cascades the direction of the flow and the characteristics at the boundaries are known. Let the subscript " $a$ " denote approaching a boundary, "b" refers to on the boundary, and "l" means leaving the boundary. For a subsonic inflow, the characteristics approaching the boundary are set equal to the characteristics on the boundary using Eq. 11:

$$
\begin{gathered}
{\left[\xi_{x}\left(\rho-\frac{p}{c_{1}^{2}}\right)\right]_{a}=\left[\xi_{x}\left(\rho-\frac{p}{c_{1}^{2}}\right)\right]_{b}} \\
{\left[\xi_{y}\left(\rho-\frac{p}{c_{1}^{2}}\right)\right]_{a}=\left[\xi_{y}\left(\rho-\frac{p}{c_{1}^{2}}\right)\right]_{b}} \\
{\left[\frac{p \nabla \xi}{\rho_{1} c_{1}}+\left(\xi_{x} u+\xi_{y} v\right)\right]_{a}=} \\
{\left[\frac{p \nabla \xi}{\rho_{1} c_{1}}+\left(\xi_{x} u+\xi_{y} v\right)\right]_{b}}
\end{gathered}
$$




$$
\begin{aligned}
& {\left[\frac{p \nabla \xi}{\rho_{1} c_{1}}-\left(\xi_{x} u+\xi_{y} v\right)\right]_{1}=} \\
& {\left[\frac{p \nabla \xi}{\rho_{1} c_{1}}-\left(\xi_{x} u+\xi_{y} v\right)\right]_{0}}
\end{aligned}
$$

Solving Eqs 13(a-d) and assuming that the metrics at the boundary are equal to the metrics at " $a$ " and "l", gives the following information for the flow variables at the inflow boundary:

$$
\begin{gathered}
p_{0}=1 / 2\left\{p_{a}+p_{1}+\right. \\
\left.\rho_{1} c_{1}\left[\xi_{x}\left(u_{a}-u_{1}\right)+\xi_{y}\left(v_{a}-v_{l}\right)\right]\right\} \\
\rho_{b}=\rho_{a}+\frac{p_{b}-p_{a}}{c_{1}^{2}} \\
u_{b}=u_{a}+\xi_{x} \frac{p_{a}-p_{b}}{\rho_{1} c_{1}} \\
v_{b}=v_{a}+\xi_{y} \frac{p_{a}-p_{b}}{\rho_{1} c_{1}}
\end{gathered}
$$

With the non-conservative flow variables known, the conservative dependant variables (Eq. 3a) can be easily computed at the boundary.

The flow variables at the outflow boundary are found in a similar manner. The characteristics on the boundary are set equal to the characteristics leaving the boundary. In this case, the incoming characteristic is the upstream running pressure wave and therefore the pressure is set equal to the pressure at "l". For internal steady flows, this is usually specified by the user. The non-conservative flow variables become:

$$
\begin{gathered}
p_{b}=p_{1} \\
\rho_{b}=\rho_{a}+\frac{p_{b}-p_{a}}{c_{2}^{2}} \\
u_{b}=u_{a}+\xi_{x} \frac{p_{a}-p_{b}}{\rho_{2} c_{2}} \\
u_{b}=v_{a}+\xi_{y} \frac{p_{a}-p_{b}}{\rho_{2} c_{2}}
\end{gathered}
$$

These characteristic variable boundary conditions are only valid for steady flows when applied to cascades since the outflow is assumed to have uniform static pressure. There are a number of flow solvers today that mistakenly use similar boundary conditions for unsteady flows. For comparison purposes, these boundary conditions will be call "reflective" boundary conditions when they are later used for unsteady flows. A better approximation for the unsteady boundary conditions are examined next.

\section{Unsteady Inflow and Outflow Boundary Conditions}

For unsteady flows in turbomachinery, the exact specification of the unsteady boundary conditions for non-linear problems is not feasible. Approximate "non-reflecting" boundary conditions are developed by assuming that linear theory can be applied. A very useful publication by Giles (Ref.21) presents the formulation of boundary conditions for internal flows. Much of the original theoretical work for this topic was done by Kreiss (Ref.22). Giles has transformed the theory into a form that can be easily implemented in general Euler solvers for turbomachinery. The basic implementation of this formulation determines the steady flow using the boundary conditions derived above and then solves the linearized Euler equations at the boundary to determine the perturbation flow variables in terms of the characteristic variables. This allows time variations of static pressure at the outflow and reduces reflections from the boundaries. $A$ brief summary, based on reference 21 is given below.

For the cases considered in this paper, $x=\xi, y=\eta$ and $t=\tau$, so that the primitive form of the Euler equations can be written as:

$$
\frac{\partial U}{\partial t}+A \frac{\partial U}{\partial x}+B \frac{\partial U}{\partial y}=0
$$

where

$$
\begin{gathered}
U=(\rho, u, v, p)^{T} \\
A=\left(\begin{array}{cccc}
u & \rho & 0 & 0 \\
0 & u & 0 & \frac{1}{\rho} \\
0 & 0 & u & 0 \\
0 & \gamma p & 0 & u
\end{array}\right) \\
B=\left(\begin{array}{cccc}
v & 0 & \rho & 0 \\
0 & v & 0 & 0 \\
0 & 0 & v & \frac{1}{\rho} \\
0 & 0 & \gamma p & v
\end{array}\right)
\end{gathered}
$$

For small-perturbations from the steady flow and 
neglecting higher order terms, a linear form of the Euler equations can be written as:

$$
\frac{\partial U^{\prime}}{\partial t}+A \frac{\partial U^{\prime}}{\partial x}+B \frac{\partial U^{\prime}}{\partial y}=0
$$

where $U^{\prime}$ are the perturbation variables given as,

$$
U^{\cdot}=(\tilde{\rho}, \tilde{u}, \tilde{v}, \tilde{p})^{\top}
$$

and $A, B$ are constant coefficient matrices based on the steady flow variables. The non-dimensional form of these matrices are:

$$
\begin{aligned}
A & =\left(\begin{array}{llll}
u & l & 0 & 0 \\
0 & u & 0 & 1 \\
0 & 0 & u & 0 \\
0 & 1 & 0 & u
\end{array}\right) \\
B & =\left(\begin{array}{llll}
v & 0 & 1 & 0 \\
0 & v & 0 & 0 \\
0 & 0 & v & 1 \\
0 & 0 & 1 & v
\end{array}\right)
\end{aligned}
$$

Following a Fourier analysis of these equations, a set of eigenvectors representing an entropy wave, a vorticity wave and upstream/downstream running pressure waves can be determined. The details of this procedure are given in reference 21 . Using these eigenvectors and assuming locally one-dimensional flow at the boundary, the characteristic variables for unsteady flows is written in terms of the perturbation variables as:

$$
\left(\begin{array}{l}
c_{1} \\
c_{2} \\
c_{3} \\
c_{4}
\end{array}\right)=\left(\begin{array}{cccc}
-1 & 0 & 0 & 1 \\
0 & 0 & 1 & 0 \\
0 & 1 & 0 & 1 \\
0 & -1 & 0 & 1
\end{array}\right)\left(\begin{array}{l}
\delta \rho \\
\delta u \\
\delta v \\
\delta p
\end{array}\right)
$$

where $\delta p, \delta u, \delta v$ and $\delta p$ are the perturbations from the steady flow.

For a subsonic inflow, the amplitudes of the incoming unsteady characteristics $\left(c_{1}, c_{2}, c_{3}\right)$ are set to zero and the outgoing characteristic ( $\left.c_{4}\right)$ is computed using Eq. 19. For subsonic outflow, $c_{4}=0$ and the remaining characteristics are computed. Once the characteristics are known, the perturbation variables are found using an inverse transform:

$$
\left(\begin{array}{c}
\delta \rho \\
\delta u \\
\delta v \\
\delta p
\end{array}\right)=\left(\begin{array}{cccc}
-1 & 0 & \frac{1}{2} & \frac{1}{2} \\
0 & 0 & \frac{1}{2} & -\frac{1}{2} \\
0 & 1 & 0 & 0 \\
0 & 0 & \frac{1}{2} & \frac{1}{2}
\end{array}\right)\left(\begin{array}{l}
c_{1} \\
c_{2} \\
c_{3} \\
c_{4}
\end{array}\right)
$$

The primitive flow variables can be found using the following relationships:

$$
\begin{aligned}
& \rho=\rho_{0}+\delta \rho \\
& u=u_{0}+\delta u \\
& v=v_{0}+\delta v \\
& p=p_{0}+\delta p
\end{aligned}
$$

where $\rho_{0}, u_{0}, v_{0}$ and $p_{0}$ are determined from the steady solution. Equation $3 \mathrm{a}$ is then used to determine the conservative flow variables at the boundaries.

\section{Results and Discussion}

A cascade of flat plates is used for studying the unsteady flow field due to small amplitude plunging motions. The purpose of the plunging motions is to propagate pressure waves upstream and downstream to study the accuracy of the CFD code and assess the resolution of the unsteady aerodynamics. While the blade vibration problem is usually associated with flutter analysis, the forcing frequencies considered will be high enough to simulate forced response problems. The small disturbances introduced by the plate motion allows for comparisons with classical linearization theory. Results are presented for various flow conditions, along with a comprehensive study of the effects of inflow/outflow boundary conditions, grid and CFL number.

\section{Model Problem}

Consider the unstaggered, flat plate cascade geometry shown in Figure 1a with a gap-to-chord ratio of one. Uniform flow with $M_{1}=0.50$ is used for the steady flow. Small plunging oscillations with $h_{1}=$ 0.001 and $\sigma=90$ degrees are used for all of the cases presented. A semi-chord reduced frequency $(k)$ of four was chosen to simulate a realistic rotor/stator frequency expected in advanced ducted propeller 
designs. According to linear theory, this flow condition propagates single mode pressure waves both upstream and downstream of the cascade. In aeroacoustic terminology, this refers to a case that is "cut-on" and in aeroelastic terminology, it is called "super-resonant." This defines a model problem with a known solution that can be used to study wave propagation using CFD.

The procedure used is to first determine a steady solution with no blade motion. For flat plates with zero incidence, this reduces to uniform flow and can be specified analytically. The unsteady solutions are started with each plate moving according to the specified interblade phase angle. Unless specified otherwise, the maximum CFL number used in each of the following solutions is unity. The numerical solution runs until the unsteady surface pressures reach periodicity. For the present study, the unsteady pressures on the plate are Fourier transformed into their real and imaginary parts after each cycle of oscillation. The solution is stopped after the coefficients from the first harmonic do not vary significantly. This usually takes anywhere from 3 to 20 cycles of plate oscillation. The solutions presented below are done on a CRAY-YMP computer and take anywhere from 20 minutes to 5 hours of CPU time, depending on the grid and time step.

\section{Visualization of the Effects of Boundary Conditions}

A set of runs are done to visually demonstrate pressure wave propagation and the importance of using correct inflow and outflow unsteady boundary conditions. Consider a uniformly spaced grid ( $\mathrm{dx}=\mathrm{dy}=.05$ chordlengths) with the inflow and outflow boundaries located six chordlengths away from the leading and trailing edges, respectively. The grid resolution is too coarse for accurate computations of surface pressures, but is sufficient for visual demonstration of pressure wave propagation to the far-field. Figure 1 (b-f) show snapshots of static pressure contours just after the plates are impulsively moved from uniform flow. The amount of time between snapshots is approximately constant and is arbitrarily denoted $\Delta T$, which gives five instantaneous plots of pressure over enough time for the downstream running wave to reach the boundary. The left block uses the "reflective" boundary conditions and the right block uses the "non-reflective" boundary conditions. Since the "reflective" boundary conditions enforce constant static pressure at the exit boundary, the downstream running wave is reflecled (Figure $1 \mathrm{e}$ and $)$. As the solution continues, the downstream domain is contaminated with an upstream-running wave that is non-physical for the desired solution. With proper grid resolution, this wave enters the cascade and affects the unsteady pressures on the plates.

Snapshots of static pressure contours are shown in Figure 2 after twenty plate oscillation cycles. There are four solutions shown; two of which are solutions using the uniform grid with the "reflective" and "non-reflective" boundary conditions shown in Figure 1. The remaining two solutions are using the "reflective" boundary conditions on grids that coarsen as they extend to the inlet and exit boundaries. Based on the contours shown in Figure $\mathbf{1}$ for the "reflective" boundary conditions, it is not surprising that the outflow region looks so contaminated compared to the "non-reflective" solutions after twenty plate oscillation cycles. Coarsening the grid introduces numerical dissipation into the solution that dampens the waves as they propagate. (This has been commonly used for isolated airfoil analysis). The grid has been coarsened such that the resolution in the passage is the same ( $\mathrm{dx}=\mathrm{dy}=.05$ chordlengths), but the aspect ratio of the cells gradually change to 3:1 $(\mathrm{dx}=.15, \mathrm{dy}=.05)$ at the inflow and outflow boundaries in one case (Figure $2 \mathrm{c}$ ) and 10:1 $(\mathrm{dx}=.50, \mathrm{dy}=.05)$ in the other case (Figure $2 \mathrm{~d})$. These figures clearly demonstrate the effect of grid coarsening, particularly for the upstream region where shorter wavelengths are being resolved with fewer grid points. Figure 3 shows the unsteady pressure distributions on the plate after the solutions have run twenty plate oscillation cycles. There is a significant difference between the "reflective" case and the "non-reflective" case using a uniform grid, as would be expected based on the contour plots in Figures 1 and 2 . Also, the solutions using the grid coarsening technique and "reflective" boundary conditions tend toward the "non-reflective" boundary conditions case using a uniform grid. It is expected that grid coarsening will only work if the grid spacing variation is smooth, as abrupt changes in the grid may cause internal reflections. 


\section{Effect of Boundary Distance From the Cascade}

An investigation of the effect of inflow and outflow boundary distance from the cascade is done using both the "reflective" and "non-reflective" boundary conditions. The upstream and downstream boundaries are placed $0.25,0.5,1.0,1.5$, and 2.0 chordlengths away from the leading and trailing edges of the plates, respectively. The grid density is refined to $\mathrm{dx}=\mathrm{dy}=.02$ chordlengths. Each case was run through 20 cycles of oscillation to give the unsteady pressure predictions shown in Figures 4 and 5. The results shown in Figure 4 use the "reflective" boundary conditions and never reach a solution that is independent of the boundary distance. In contrast, the cases using the "non-reflective" boundary conditions (Figure 5) are nearly identical when the boundaries are 1.5 and 2.0 chordlengths away from the cascade. This demonstrates the advantage of using "non-reflective" boundary conditions for reducing the computational domain.

Results from linear theory (ref.1) are also plotted in Figures 4 and 5. The results using the "non-reflective" boundary conditions that are independent of the boundary distance are in general, but not exact agreement with the theory (short dashed lines in Figure 5). Since the theory is exact for flat plates, the differences between the solutions are due to numerical error. It will be demonstrated later that the discrepancies are due to grid resolution. For now, the study on the effect of boundary distance using different inflow and outflow boundary conditions should still be valid.

\section{Effect of Boundary Conditions With Grid Coarsening}

The results shown in Figures 2 and 3 have already demonstrated the effects of grid coarsening using the "reflective" boundary conditions on a coarse grid. Based on these results, damping the pressure waves by coarsening the grid should render a solution insensitive to either the "reflective" or "non-reflective" boundary conditions. To verify this, another set of solutions are considered using a finer grid near the plates that smoothly coarsens to the inflow and outhlow boundaries. The same boundary distances of six chordlengths upstream and downstream are used. The grid density is $\mathrm{dx}=\mathrm{dy}=.02$ chordlengths in the passage and coarsens to $\mathrm{dx}=.50$ and $\mathrm{dy}=.02$ chordlengths at the inflow and outflow boundaries. The unsteady pressure distributions are plotted in Figure 6 for solutions using both the "reflective" and "non-reflective" boundary conditions, along with the exact solution from linear theory. The solutions are identical with each other and differ from the theory by the same amount found in Figure 5 using the "non-reflective" boundary conditions. Therefore, a solution has been found that is not being influenced by reflections from the inflow and outflow boundaries.

\section{Effect of Time Step (CFL Number)}

All of the previous solutions were obtained using a maximum CFL number of unity. For cases with varying grid spacing, this means that the smallest cell does not exceed a CFL number of one and the larger cells have a CFL number less than one. The grids with uniform spacing have a CFL number of one for every cell. Specifying a maximum CFL number of one preserves, at least formally, the second-order temporal accuracy of the numerical solution. However, many unsteady solutions using a time-marching code run higher CFL numbers to reduce the run times. When this is done, an assumption is made that the higher CFL numbers occur only at the smaller cells and do not significantly effect the global solution. The following solutions show how bad this assumption can be if care is not taken to ensure that the CFL number is small enough to resolve the unsteady flow field.

A study of the effects of CFL number is done on two types of grids; a uniformly spaced grid and a grid with points clustered near the plates. The "non-reflecting" boundary conditions are used in all of the remaining results. The uniformly spaced grid $(\mathrm{dx}=\mathrm{dy}=.02$ chordlengths) is identical to the one used in the study of boundary distance effects with the boundary located 0.5 chordlengths away from the cascade (Figures 4 and 5). Unsteady pressure predictions using three different values of CFL number are shown in Figure 7. The results using a CFL number of one are the same as those shown in Figure 5. The good agreement with linear theory is fortuitous since the predictions with a boundary distance of 0.5 chordlengths was found to be insufficient (see discussion for Figure 5). Increasing the CFL number to values of two and three introduces temporal error, as expected. Using a CFL number of three completely destroys the character of the unsteady 
wave forms. For this case, two solutions have been plotted after twenty and forty cycles of plate oscillation to verify that the solution has reached periodicity.

A similar study is shown in Figure 8 for a grid that is clustered in the $y$-direction. The spacing near the plates is $\mathrm{dx}=.05, \mathrm{dy}=.02$ chordlengths and smoothly varies to a spacing of $\mathrm{dx}=\mathrm{dy}=.05$ chordlengths at the center of the passage. Hence, the maximum CFL number occurs near the plates. The boundary distance is 0.5 chordlengths upstream and downstream of the cascade. From the predictions shown in Figure 8, clustering the grid near the surface has helped preserve the character of the pressure waves, but still quickly loses the accuracy for maximum CFL numbers of two and three. It should be noted that for a maximum CFL number of three, the minimum CFL number is 0.4 on this grid. This means that there is a portion of the grid that should convect disturbances correctly near the center of the passage. However, in the region of the grid near the plates, which is where the unsteady disturbances are being generated, the correct magnitude and phase are not being computed. This is why the nature of the disturbance is captured with a clustered grid, but the accuracy is poor for higher CFL numbers. As demonstrated in the next section, it is possible for highly clustered grids to do a reasonable job of predicting the unsteady flow field providing that an adequate portion of the grid has a CFL number less than unity.

\section{Effects of Grid Density}

Based on the studies presented above, it appears that a clustered grid with a small CFL number is needed to resolve the unsteady pressure waves. However, running a maximum CFL number of unity on a highly clustered grid would require extremely large amounts of computational time. Therefore, it is desirable to determine a balance between grid clustering and maximum CFL number. Three grid densities $(59 \times 21$, $121 \times 41,244 \times 81$ ) with clustering near the leading and trailing edges $(\mathrm{dx}=.003, \mathrm{dy}=.001$ chordlengths) are chosen to see if the correct magnitude and phase of the unsteady pressure waves can be computed. The grids are stretched six chordlengths upstream and downstream of the cascade using the grid coarsening technique. A maximum CFL number of ten is tried, which occurs near the leading and trailing edges of the plates. The CFL number quickly diminishes to less than one away from the plates, leaving a large percentage of the grid with an adequate time step to resolve the unsteady flow. The stagger angle has been changed from 0 to 5 degrees for reasons discussed in the next section. Comparisons of the unsteady pressure distributions are presented in Figure 9 and show excellent agreement with linear theory for grid densities of $121 \times 41$ and $244 \times 81$. It appears that the $121 \times 41$ grid is sufficient for this case. Also, clustering the grid near the plates is essential for accuracy (ie. agreement with linear theory). Obviously, it is not possible to determine an optimal maximum CFL number for a specified grid distribution since they are highly coupled and depend on the nature of the unsteady flow field. However, it is clear that each case being considered needs to be tried on more than one grid with various levels of clustering and at least two CFL numbers per grid. (The message here is that what works for one set of flow conditions will not necessarily work for another set of flow conditions - a point that seems obvious, but is often overlooked.)

\section{Effects of Stagger Angle}

The results shown for zero degree stagger have not been in as good agreement with linear theory as expected. A study of the effects of stagger angle on the numerical solution are shown in Figure 10 using the best grid distribution presented in the previous section $(121 \times 41)$. Three stagger angles $(0,5$, and 45 degrees) are presented and compared with linear theory. By varying the stagger angle, the direction and wavelength of the single mode pressure waves propagating upstream and downstream are different. This tests the numerical solutions for waves propagating through the grid cells from different directions. The results show that the staggered results are in good agreement with the theory, while the unstaggered case shows small deviations near the forward portion of the plate. It is not clear why the results with zero degree stagger deviate from the theory and why a small change in the stagger angle (5 degrees) significantly improves the predictions. However, the results for staggered cascades shows that good agreement with theory can be obtained for cases with different directions of pressure wave propagation. 


\section{Effects of Oscillation Frequency}

Finally, the effects of increasing the oscillation frequency are determined by investigating values of $k$ $=6$ and $k=8$. Increasing the frequency causes higher modes to propagate from the cascade, as determined by linear theory. For all of the previous cases with $k=4$, single mode pressure waves propagate upstream and downstream of the cascade (cut-on for the fundamental wave number). If the frequency of oscillation is increased to say $k=6$, then two modes propagate both upstream and downstream, which corresponds to the fundamental mode and its next harmonic. Similarly, increasing the reduced frequency to 8 causes three modes propagate. Increasing the oscillation frequency is an easy way to systematically add modes to the flow field for studying multi-mode, pressure wave propagation. The higher frequencies and higher modes introduce shorter wavelengths and requires finer grid resolution. Based on the results in Figure 9, resolving the higher modes will require a finer grid since the $121 \times 41$ grid was just fine enough to resolve the single-mode wavelengths. Figure 11 shows the predictions for three reduced frequencies and two grid sizes (121 x 41 and $324 \times 81)$. As expected, the higher oscillation frequencies require finer grid resolution. In fact, the $324 \times 81$ grid is still not fine enough to resolve the waves near the forward portion of the plate when $k=8$. This demonstrates how difficult it can be to model unsteady flow fields that require accurate solutions for the higher harmonics.

\section{Conclusions}

An investigation of the unsteady flow field around an oscillating cascade of flat plates with zero stagger is done using a time-marching Euler code. The study demonstrates the importance of using proper unsteady boundary conditions, grid resolution and time step size to accurately model the unsteady flow field. Imposing constant static pressure across the outflow causes reflections from the downstream boundary and is shown by plotting pressure contours at various times. These reflections contaminate the unsteady pressure distributions on the plates. A study on the effect of boundary distance shows that the unsteady plate pressures never reach a solution that is independent of boundary distance when reflective boundary conditions are used. Using approximate non-reflecting boundary conditions based on linear theory improves the solutions and allows the boundaries to be placed closer to the cascade (approximately 1.5 to 2 chordlengths away from the cascade). Coarsening the grid by stretching the boundaries away from the cascade introduces numerical damping into the computational domain and is also an effective way to reduce reflections from the boundaries, although this method can only be used when simulating blade motion for a single blade row since all disturbances are damped. These solutions are insensitive to "reflecting" or "non-reflecting" boundary conditions.

Several solutions are presented that vary the time step size on both a clustered grid and a uniformly spaced grid. The results show that the unsteady flow field becomes significantly inaccurate as the CFL number exceeds one on a uniformly spaced grid. The clustered grid results do a better job of capturing the flow, but still become inaccurate as the CFL number is increased. Good agreement with linear theory is found on highly clustered grids that stretch six chordlengths upstream and downstream from the cascade (grid coarsening). For these cases, the maximum CFL number was ten and quickly diminished to less than one away from the plates.

Predictions for various stagger angles and plate oscillation frequencies show good agreement with linear theory as long as the grid is properly resolved and the CFL number is sufficiently small. Since the wave characteristics vary with different flow conditions, it is difficult to generalize CFL number and grid resoluton requirements. The results presented in this paper should serve as a guide for determining initial guesses for these requirements, but they also demonstrate the importance of investigating more than one run for each flow condition to validate numerical solutions.

\section{Acknowledgments}

The author would like to thank Dr. Timothy Swafford, Dr. David Whitfield and Dr. Mark Janus, of Mississippi State University, for the helpful discussions concerning the flow algorithm. Also, Dr. John Adamczyk, of NASA Lewis Research Center, for his encouragement during this investigation. 


\section{References}

1. Smith, S.N., "Discrete Frequency Sound Generation in Axial Flow Turbomachines," ARC-R/M-3709, 1971.

2. Whitehead, D.S., "Classical Two-Dimensional Methods," AGARD Manual on Acroelasticity in Axial-Flow Turbomachines Volume 1 Unsteady Turbomachinery Aerodynamics, AGARDograph No. 298, M.F. Platzer and F.O. Carta, editors, London, 1987, pp. (3-1) - (3-30).

3. Verdon, J.M. and Casper, J.R., "A Linearized Unsteady Aerodynamic Analysis for Transonic Cascades," Joumal of Fluid Mechanics, Vol. 149, December 1984, pp. 403-429.

4. Hall, K.C. and Crawley, E.F., "Calculation of Unsteady Flows in Turbomachinery Using the Linearized Euler Equations," Fourth Symposium on Unsteady Aerodynamics and Aeroelasticity of Turbomachines and Propellers, Aachen, West Germany, September 1987.

5. Fang, J. and Atassi, H.M., "Compressible Flows with Vortical Disturbances Around a Cascade of Loaded Airfoils," Sixth Symposium on Unsteady Aerodynamics and Aeroclasticity of Turbomachines and Propellers, University of Notre Dame, U.S.A., September 1991.

6. Kao, Y.F., "A Two-Dimensional Unsteady Analysis for Transonic and Supersonic Cascade Flows," Ph.D. Thesis, Purduc University, May 1989.

7. Fransson, T.H. and Pandolfi, M., "Numerical Investigation of Unsteady Subsonic Compressible Flows Through an Oscillating Cascade," ASME Paper 86-GT-304, June 1986.

8. Huff, D.L., "Numerical Analysis of Flow Through Oscillating Cascade Sections," AIAA Paper 89-0437, Jan. 1989.

9. He, L., "An Euler Solution for Unsteady Flows Around Oscillating Blades," ASME Paper 89-GT-279, June 1989.

10. Gerolymos, G.A., Blin, E., Quiniou, H., "Comparison of Inviscid Computations with Theory and Experiment in Vibrating Transonic Compressor Cascades," ASME Paper 90-GT-373, June 1990.

11. Giles, M. and Haimes, R., "Validation of a Numerical Method for Unsteady Flow Calculations" ASME Paper 91-GT-271, June 1991.
12. Huff, D.L., Swafford, T.W. And Reddy, T.S.R., "Eulcr Flow Predictions for an Oscillating Cascade Using a High Resolution Wave-Split Scheme," ASME Paper 91-GT-198, June 1991.

13. Whitfield, D.L., Janus, J.M., and Simpson, L.B., "Implicit Finite Volume High Resolution Wave-Split Scheme for Solving the Unsteady Three-Dimensional Euler and Navier-Stokes Equations on Stationary or Dynamic Grids," Mississippi State Engineering and Industrial Research Station Report No. MSSU-EIRS-ASE-88-2, February, 1988.

14. Prewitt, Nathan C., "Two-Dimensional Euler Code for the Prediction of Pressure Distributions About an Airfoil,"MS Thesis, Mississippi State University, Mississippi State, MS, December 1988.

15. Janus, J.M., "Advanced 3-D CFD Algorithm for Turbomachinery," PhD Dissertation, Mississippi State University, Mississippi State, MS, May 1989.

16. Arabshahi, A., "A Dynamic Multiblock Approach to Solving the Unsteady Euler Equations About Complex Configurations," PhD Dissertation, Mississippi State University, Mississippi State, MS, May 1989.

17. Whitfield, D.L., Janus, J.M., and Arabshahi, A., "Unsteady Euler Solutions on Dynamic Blocked Grids for Complex Configurations," AGARD Conference Proceedings, No.464, 1990.

18. Roc, P.L., "Approximate Rieman Solvers, Parameter Vectors, and Difference Schemes," Joumal of Computational Physics, Vol. 43, pp. 357-372, 1981.

19. Beach, T.A., "An Interactive Grid Generation Procedure for Axial and Radial Flow Turbomachinery," AIAA Paper 90-0344, 1990.

20. Janus, J.M., "The Development of A Three-Dimensional Split Flux Vector Solver with Dynamic Grid Applications," MS Thesis, Mississippi State University, Mississippi State, MS, August 1984.

21. Giles, M., "Non-Reflecting Boundary Conditions for Euler Equation Calculations," AIAA Paper 89-1942-CP, 1989.

22. Kreiss, H.O., "Initial Boundary Value Problems for Hyperbolic Systems," Communications on Pure and Applied Mathematics, 23:277-298, 1970. 


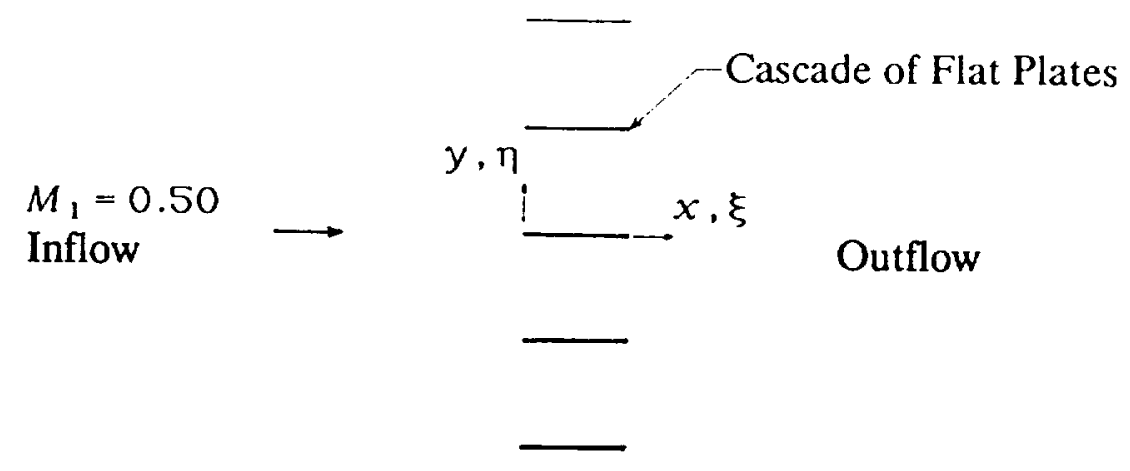

a) Cascade geometry for model problem

Reflective

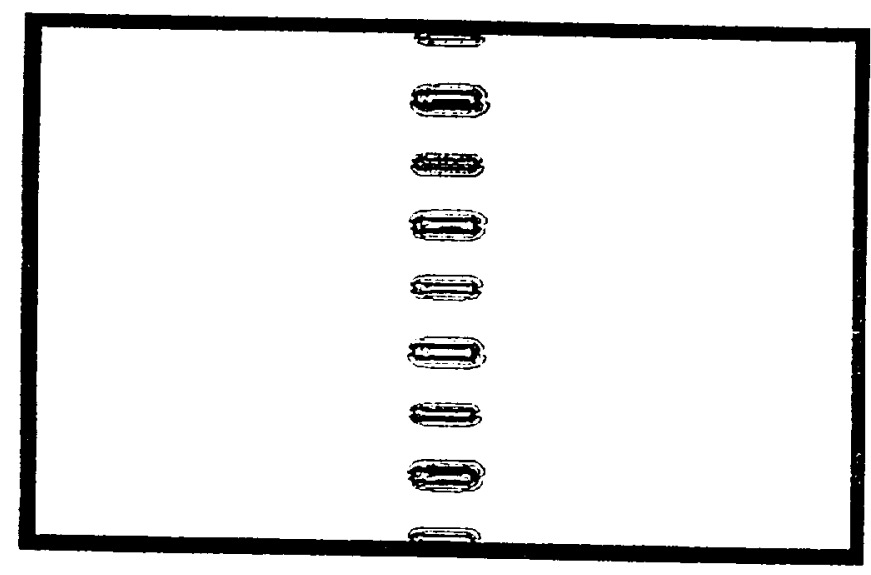

Non-Reflective

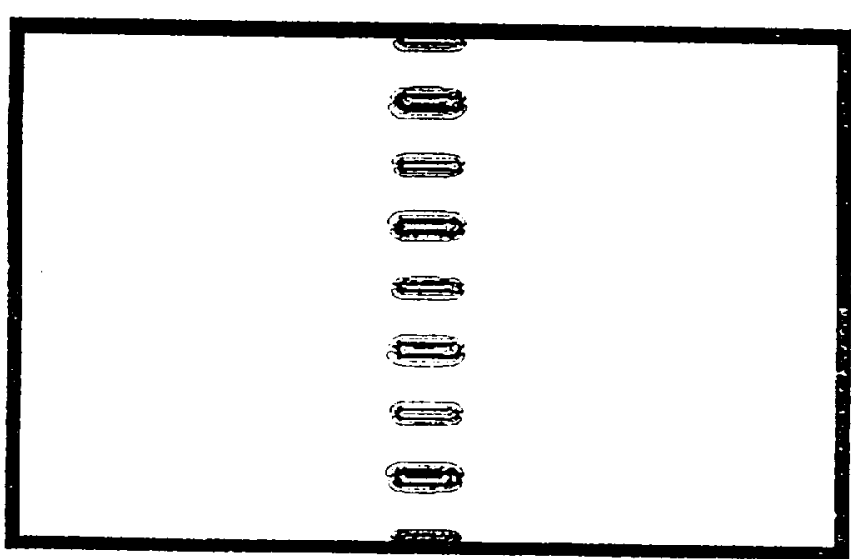

Reflective

b) $t>0$

Non-Reflective
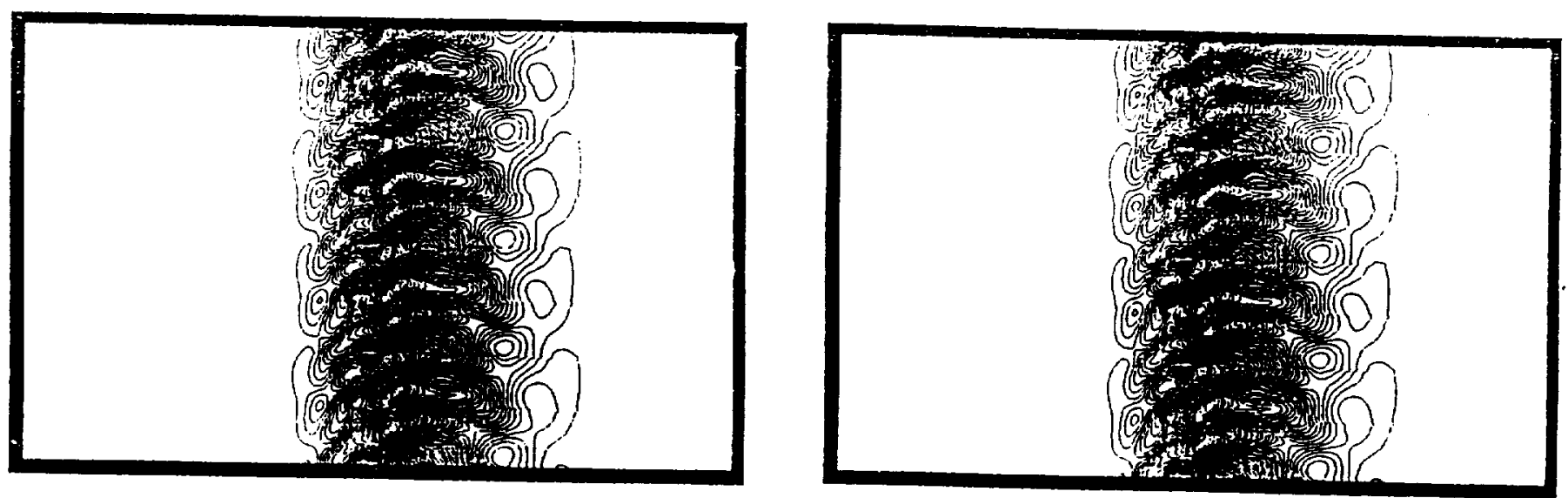

c) $t=\Delta T$

Figure 1. Cascade geometry and instantaneous static pressures for model problem. 
Reflective

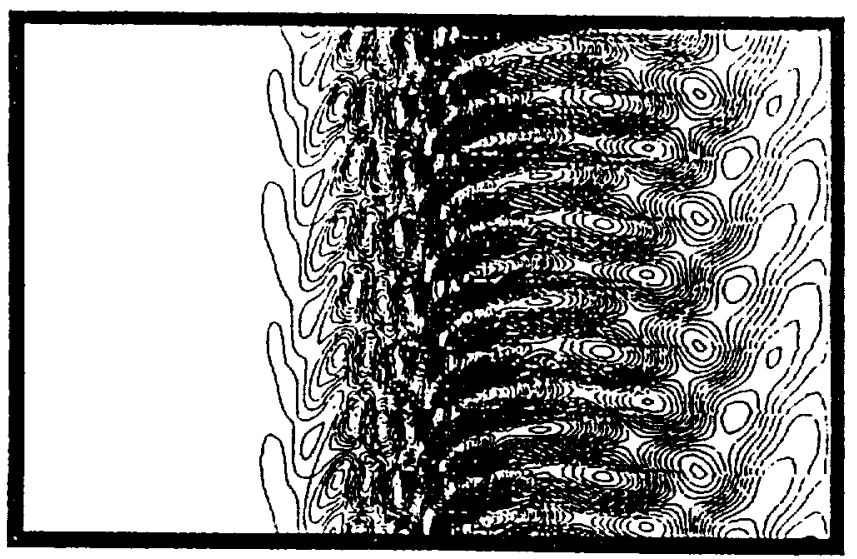

Non-Reflective

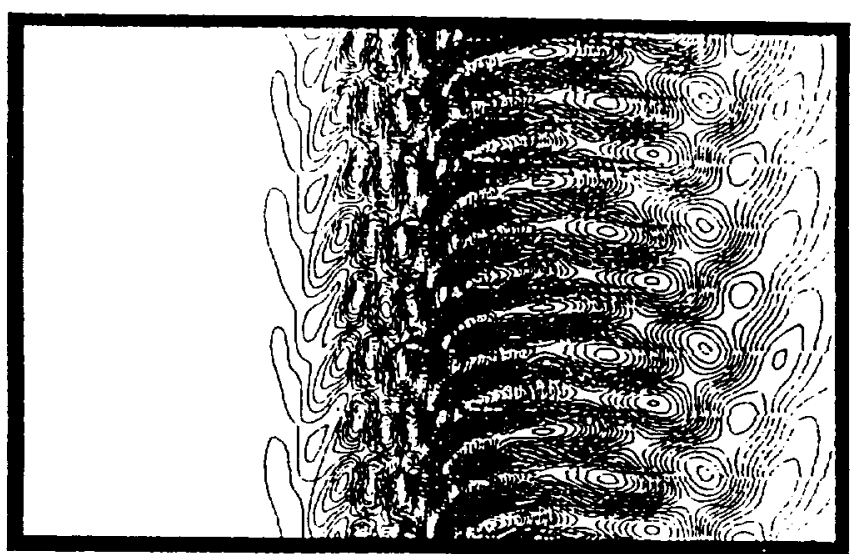

d) $t=2 \Delta T$

\section{Reflective}

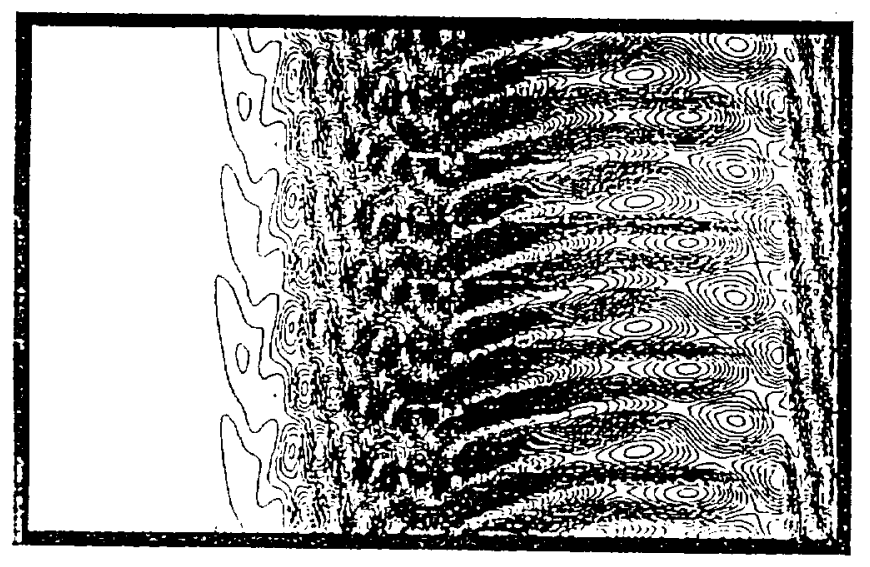

Non-Reflective

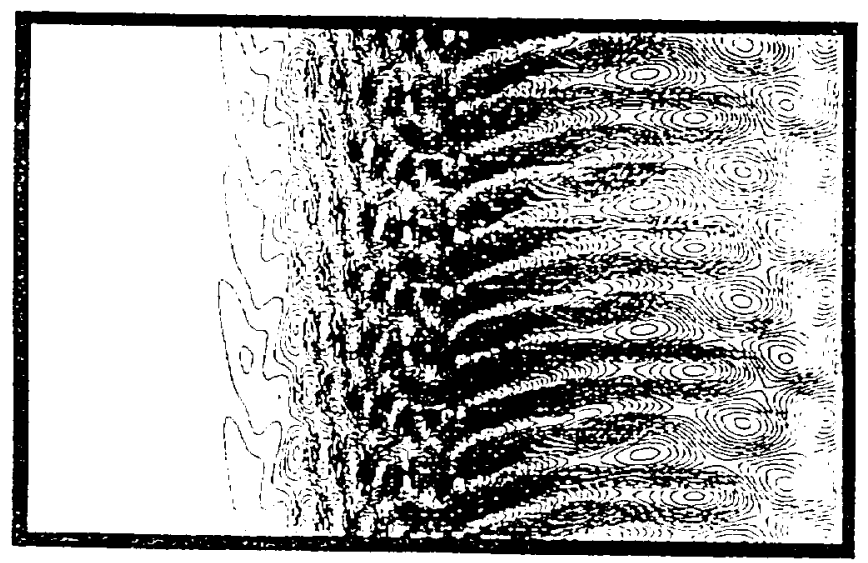

e) $t=3 \triangle T$
Reflective

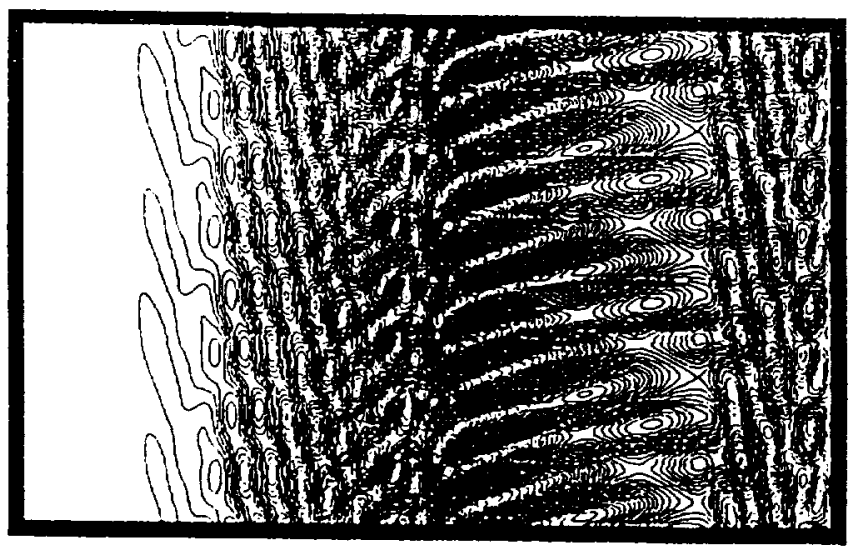

Non-Reflective

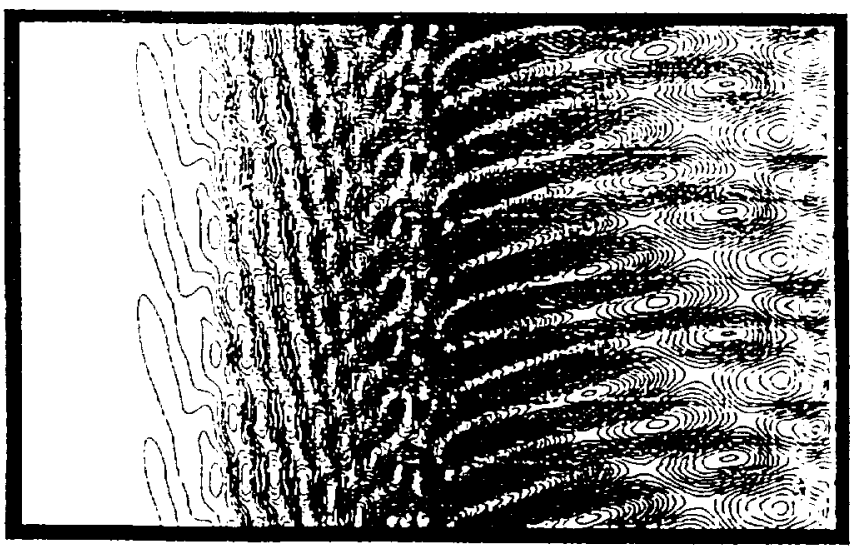

f) $t=4 \Delta T$

Figure 1. (cont.) Cascade geometry and instantaneous static pressures for model problem. 


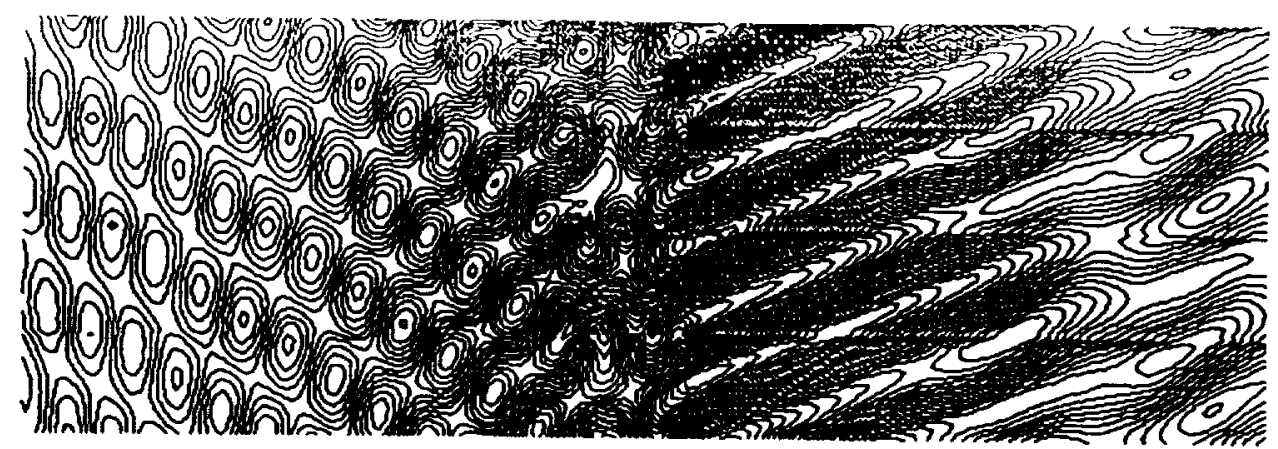

a) Non-reflective boundary conditions, uniform grid.

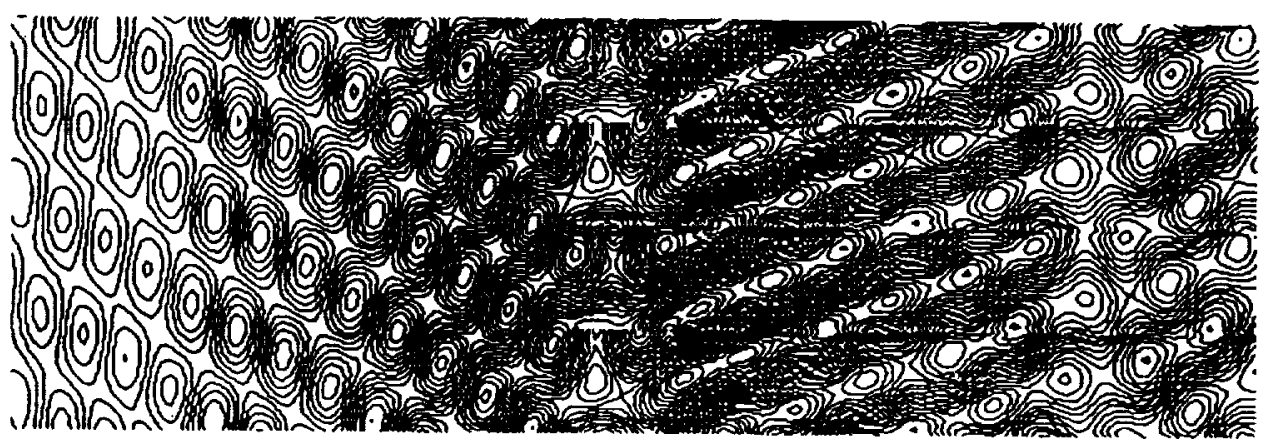

b) Reflective boundary conditions, uniform grid.

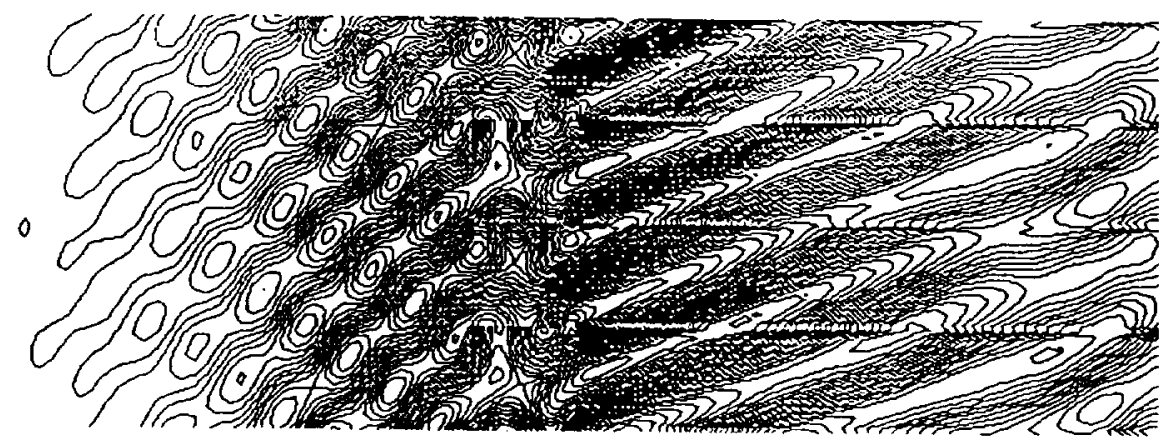

c) Reflective boundary conditions, grid coarsening 3:1.

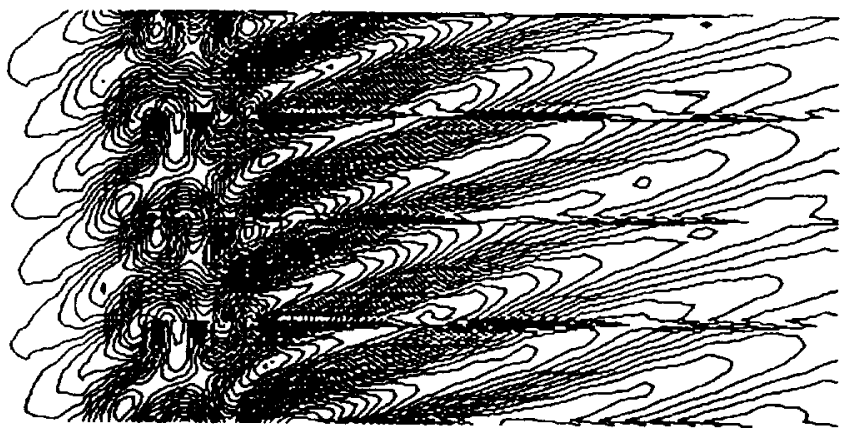

d) Reflective boundary conditions, grid coarsening 10:1.

Figure 2. Static pressure contours after twenty cycles of plate oscillations. 


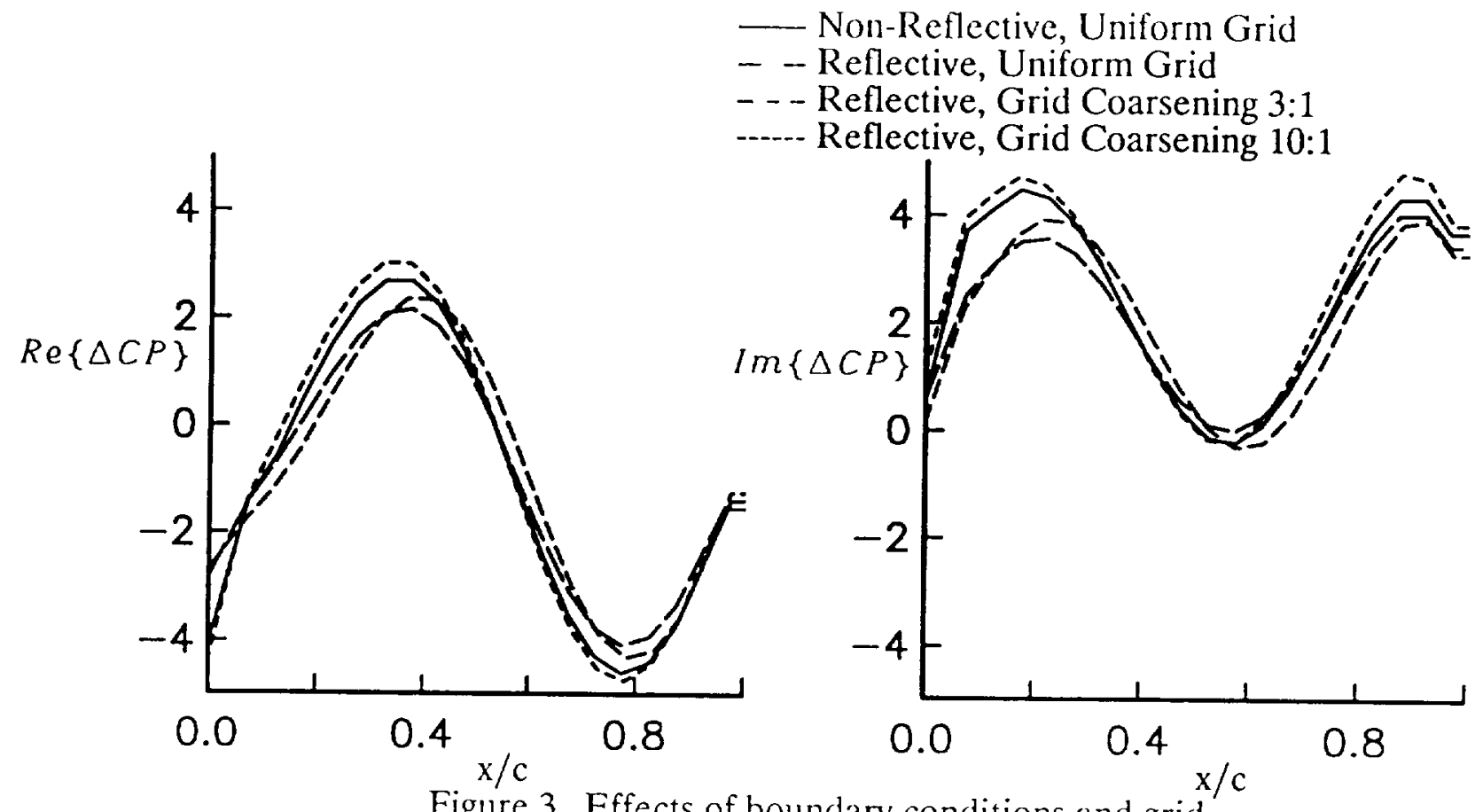

Figure 3. Effects of boundary conditions and grid on unsteady pressure distributions.

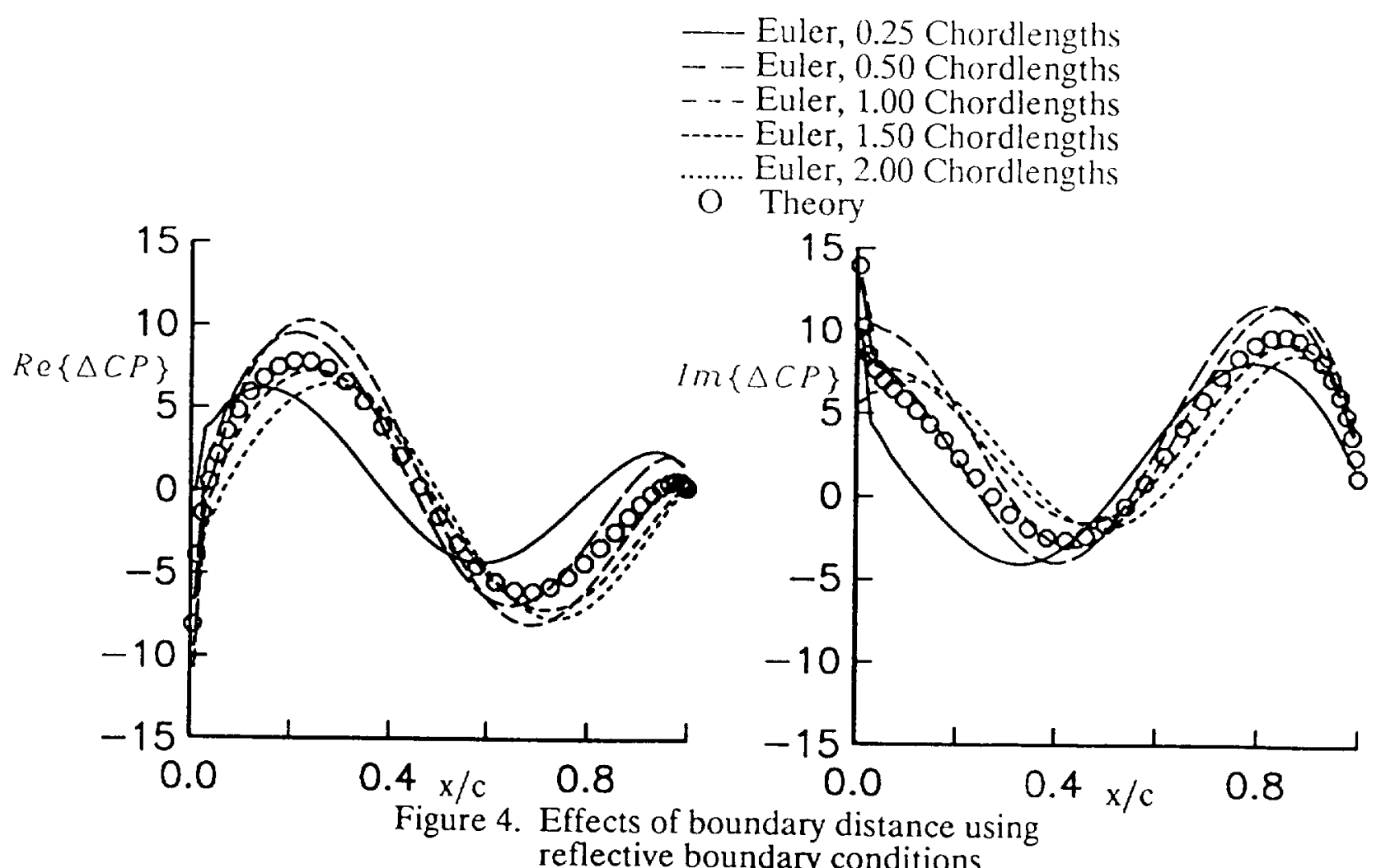




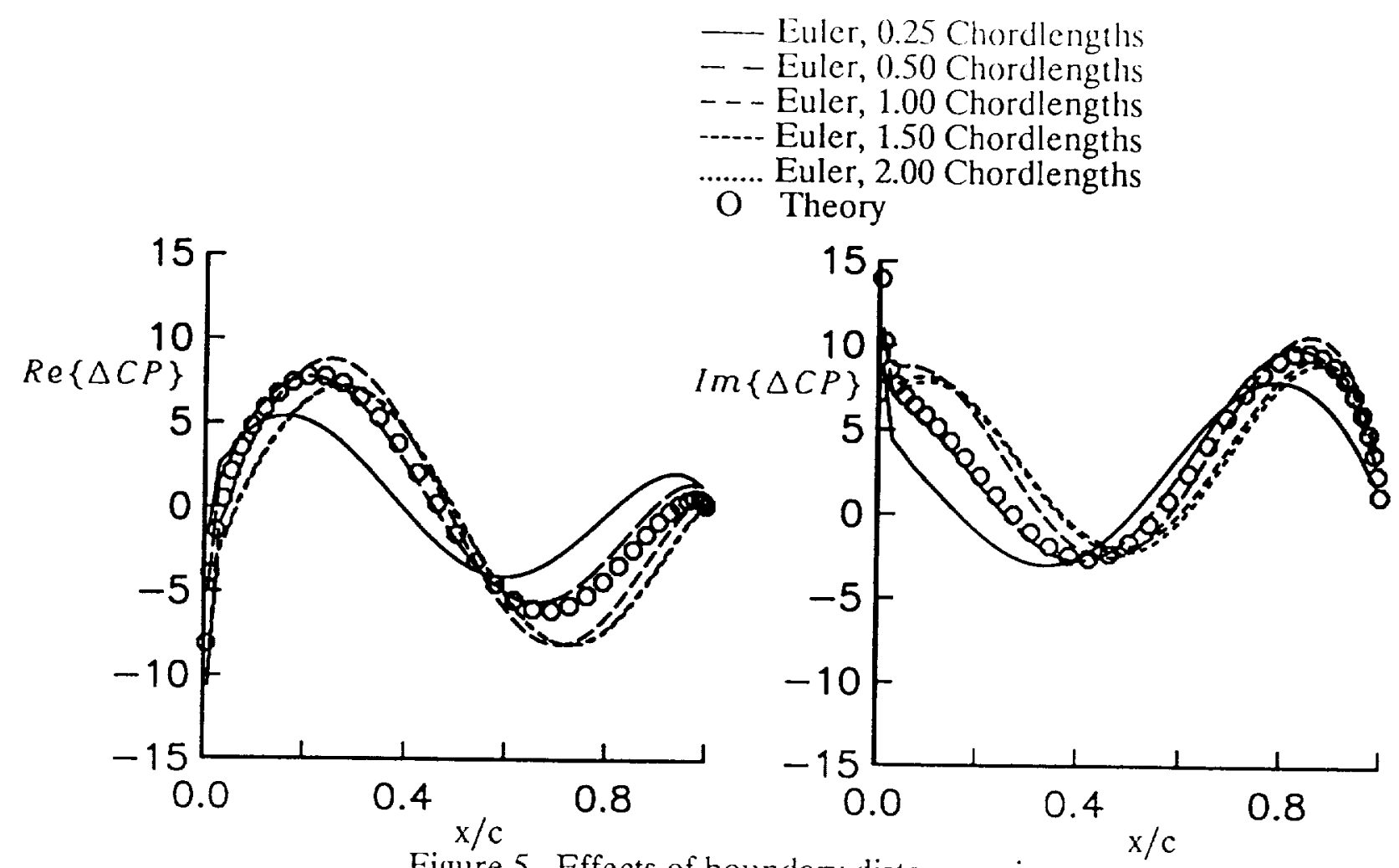

Figure 5. Effects of boundary distance using non-reflective boundary conditions.

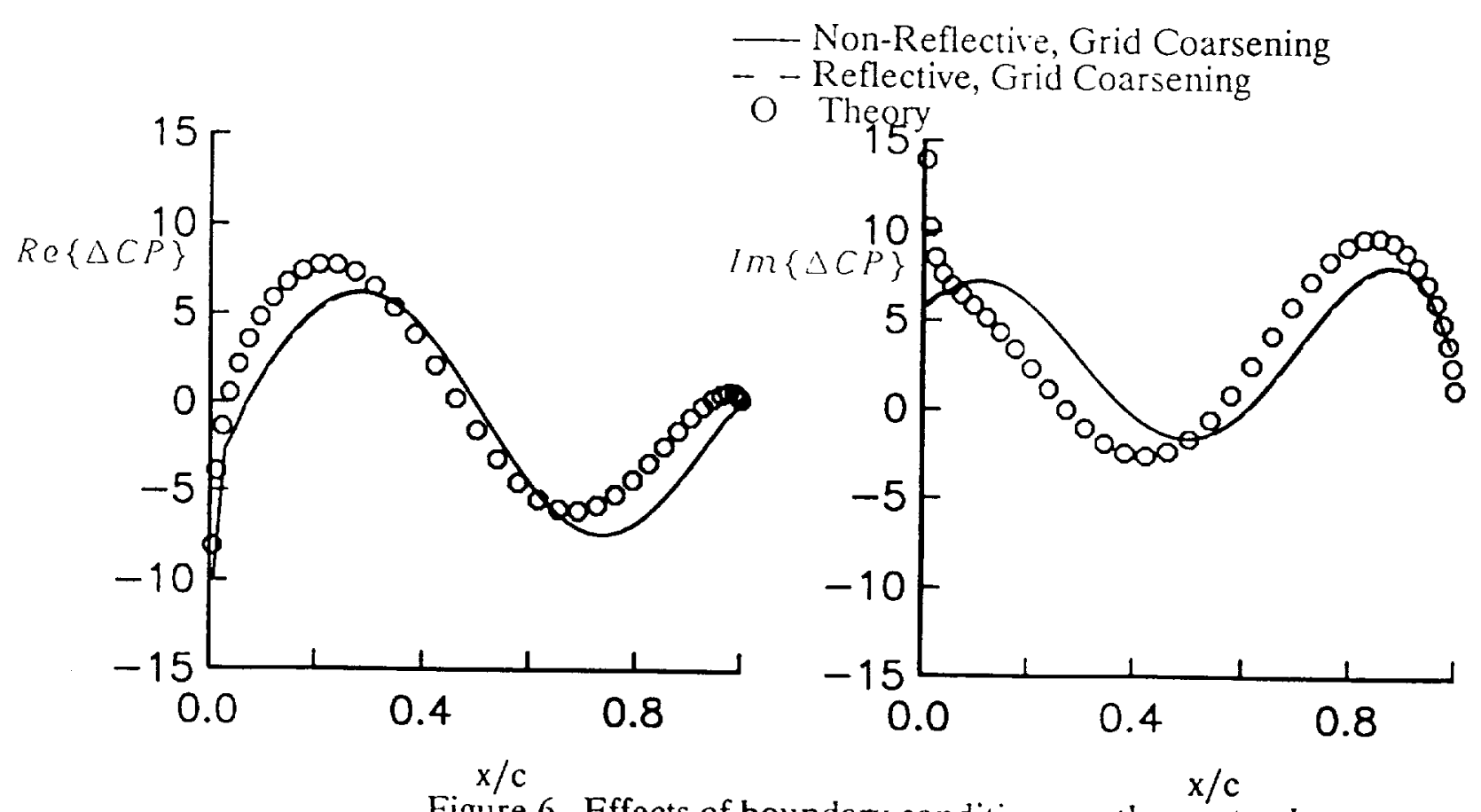

Figure 6 . Effects of boundary conditions on the unsteady pressure distributions using grid coarsening. 


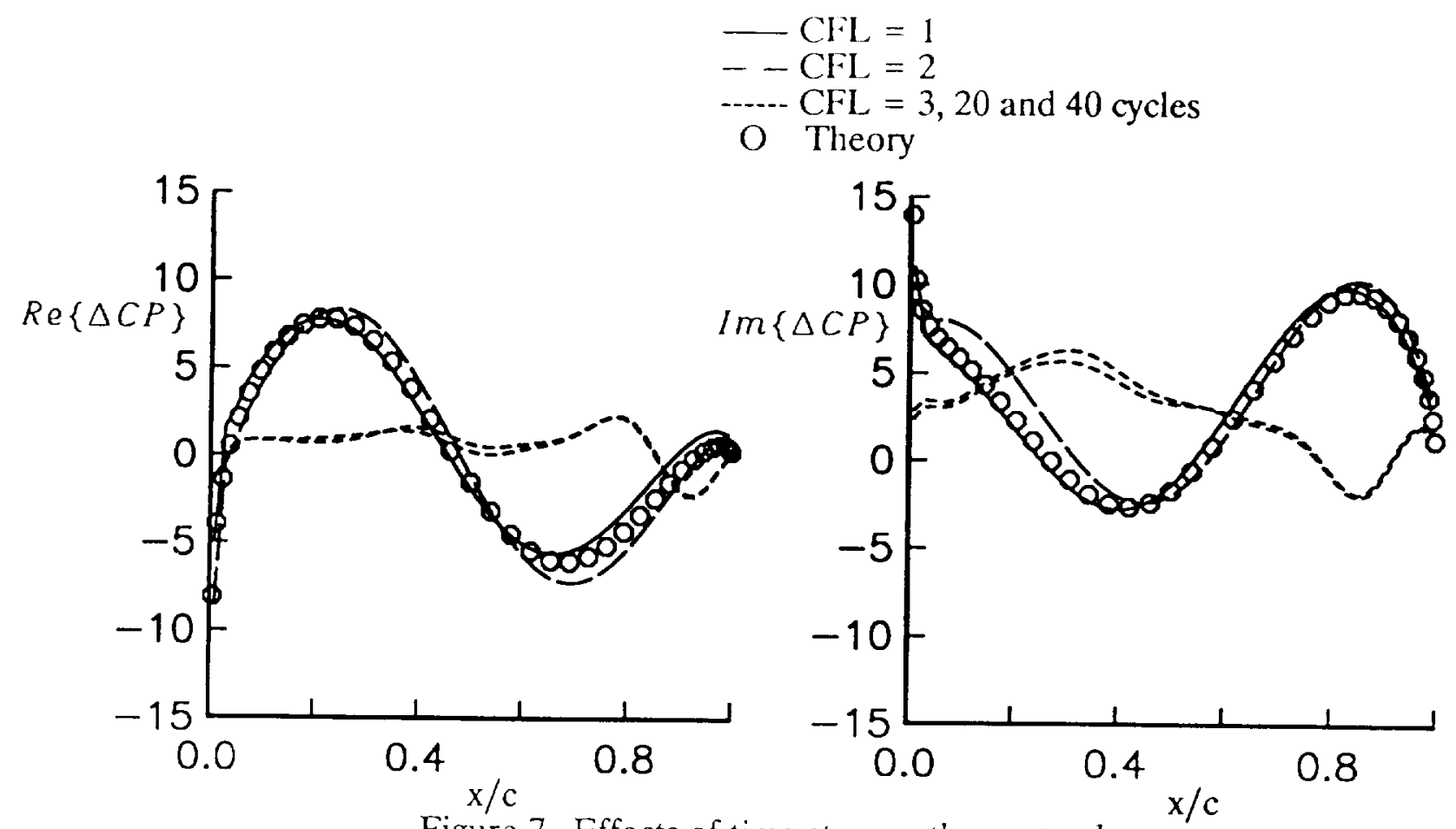

Figure 7. Effects of time step on the unsteady pressure distributions using a uniform grid.

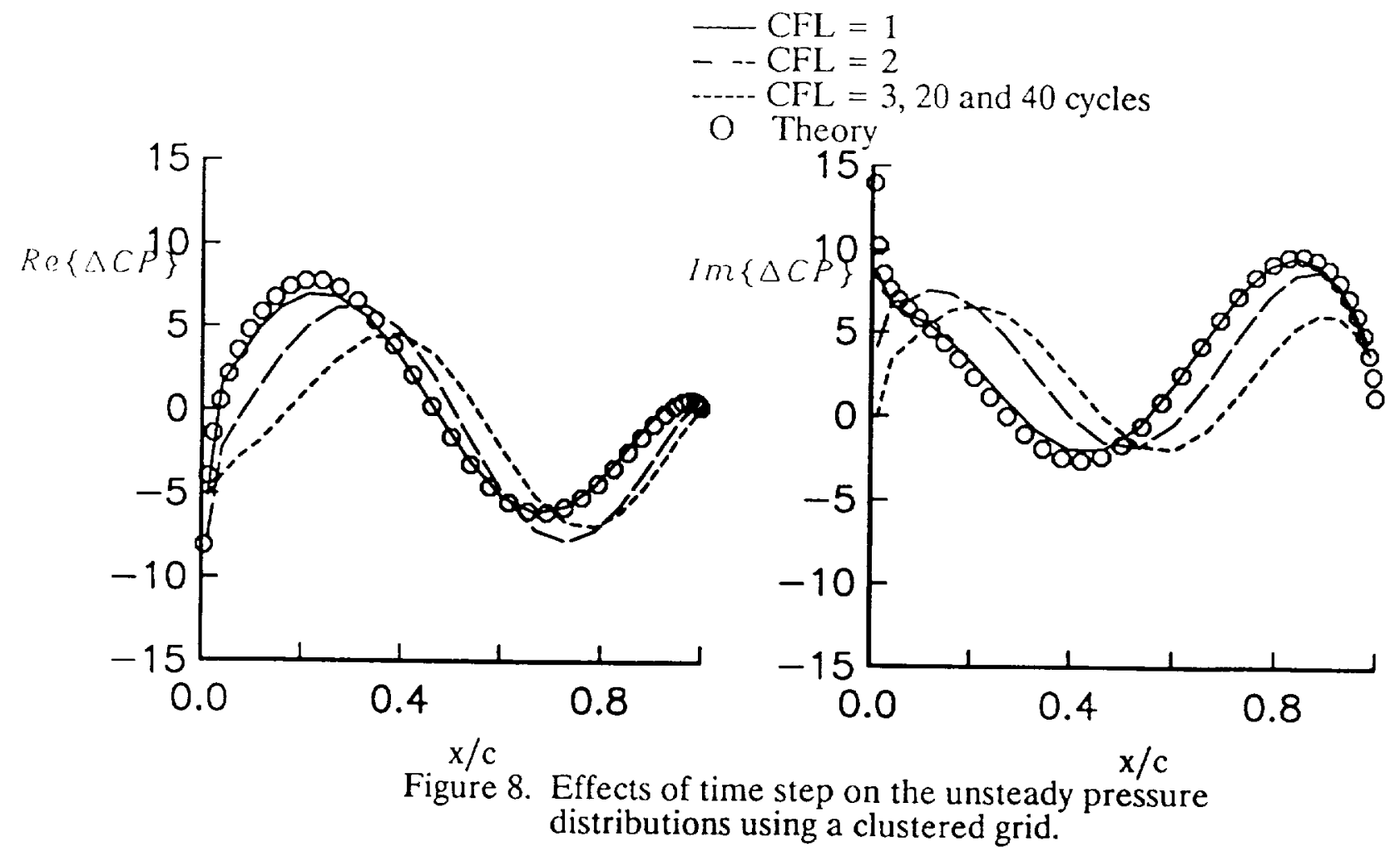




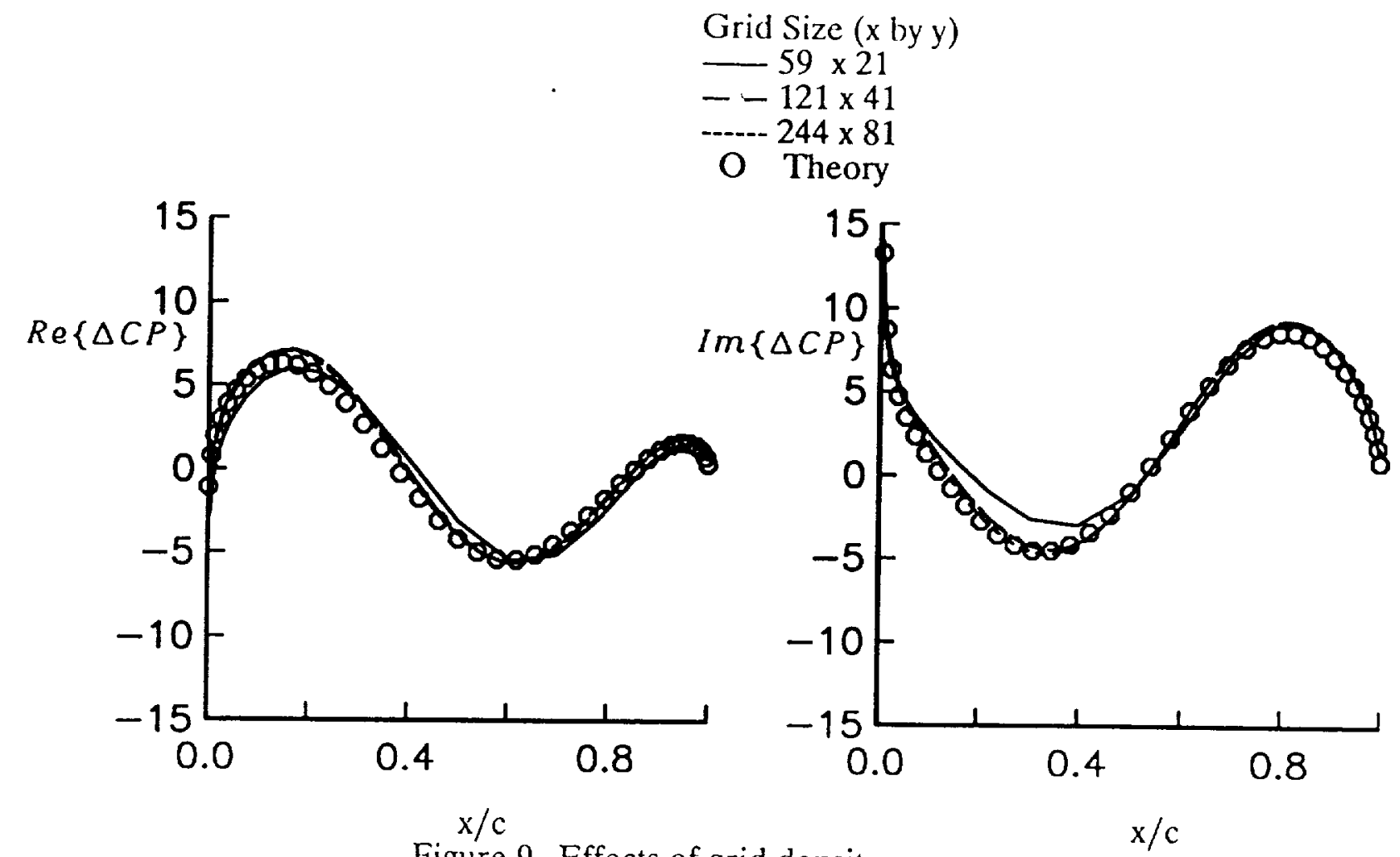

Figure 9. Effects of grid density.

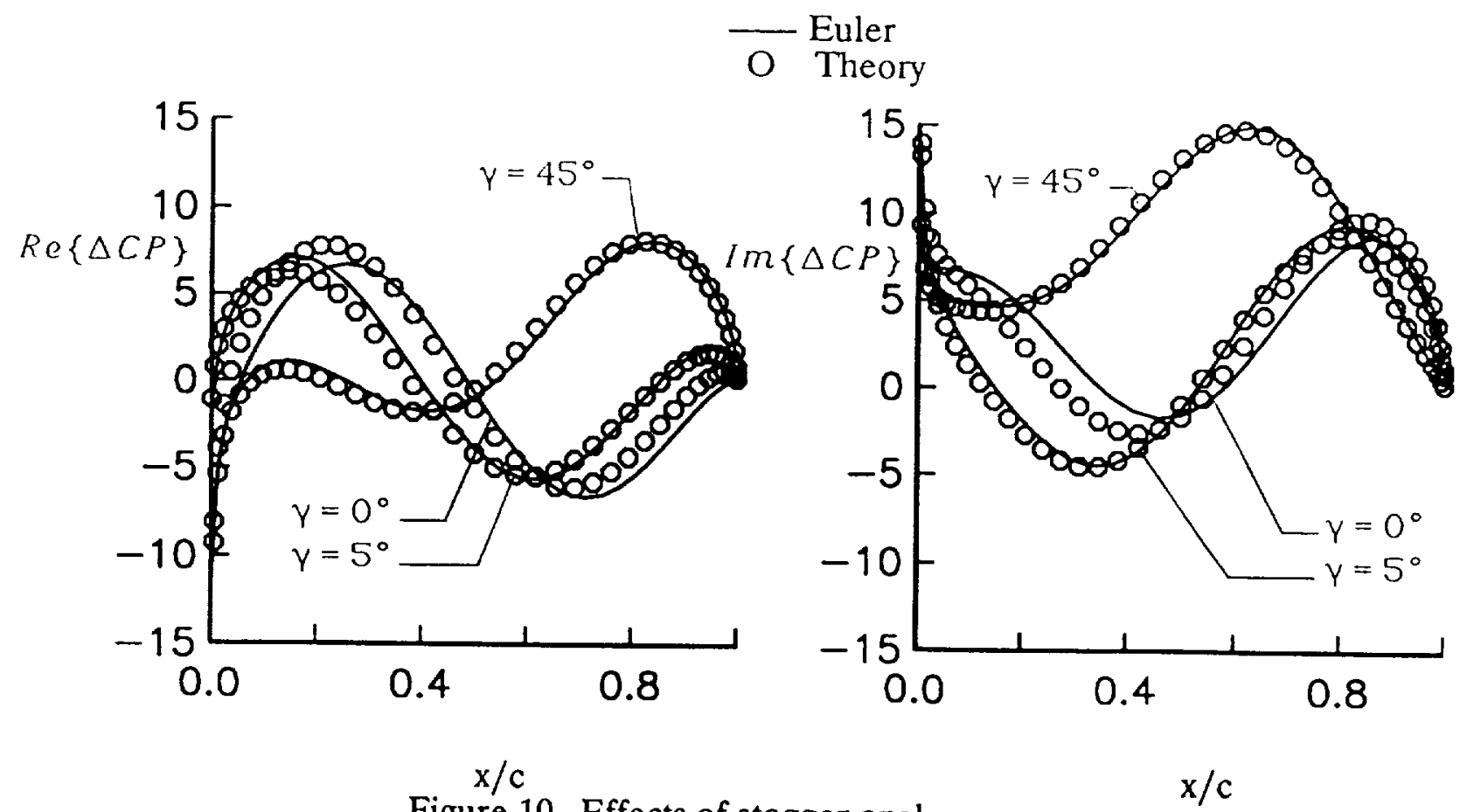

Figure 10. Effects of stagger angle. 


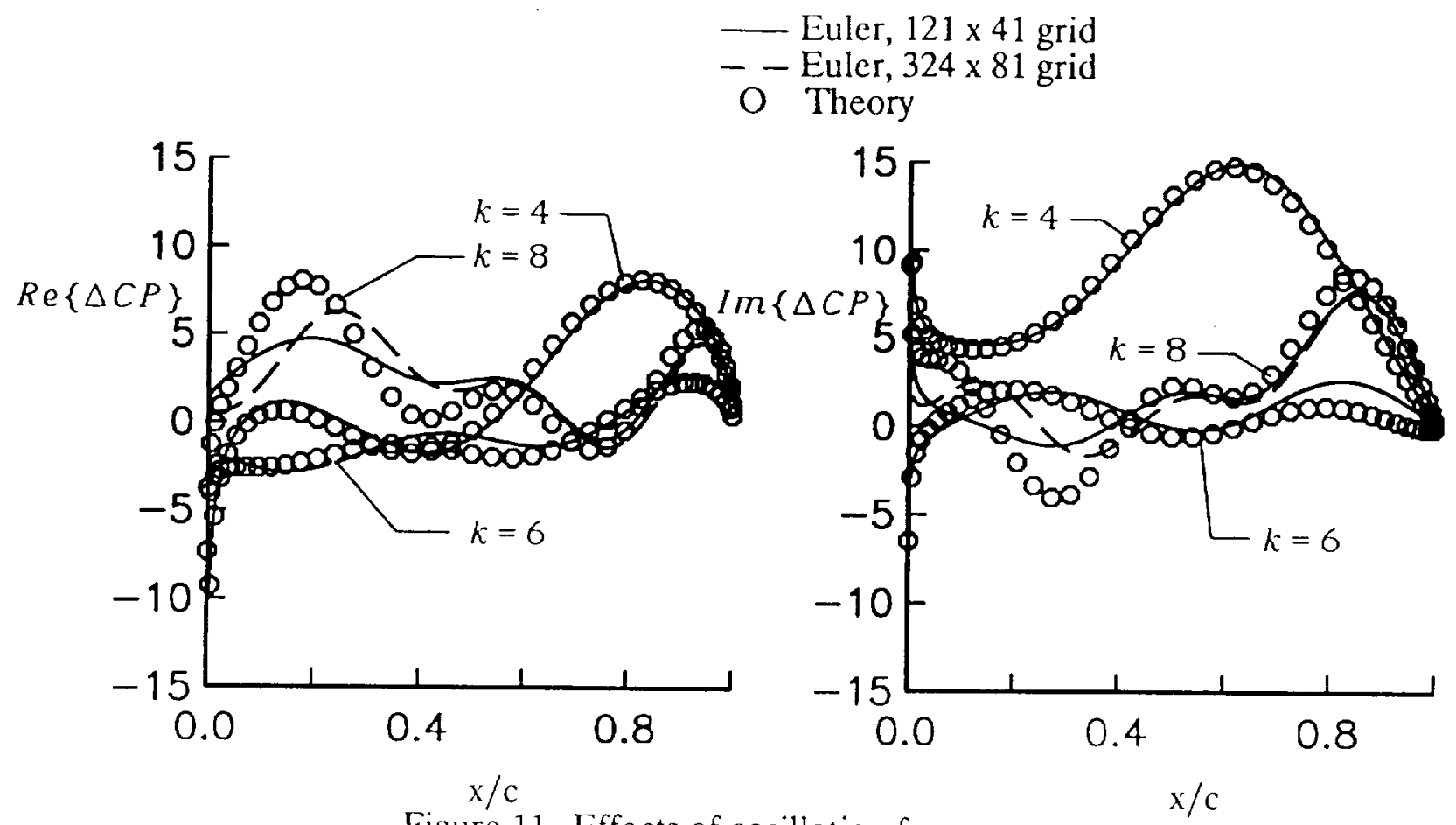

Figure 11. Effects of oscillation frequency. 
Public reporting burden for this collection of information is estimated to average 1 hour per response. including the time for reviewing instructions, searching existing data sources, gathering and maintaining the data needed, and completing and reviewing the collection of information. Send comments regarding this burden estimate or any other aspect of this Davis Highway, Suite 1204, Arlington, VA 22202-4302, and to the Office of Management and Budget, Paperwork Reduction Project (0704-0188), Washington, DC 20503.
20

\begin{tabular}{|l|l|l}
\hline 1. AGENCY USE ONLY (Leave blank) & 2. REPORT DATE & $\begin{array}{r}\text { R. REPORT TYPE AND DATES COVERED } \\
\text { Technical Memorandum }\end{array}$ \\
\hline
\end{tabular}

4. TITLE AND SUBTITLE

5. FUNDING NUMBERS

Pressure Wave Propagation Studies for Oscillating Cascades

6. AUTHOR(S)

Dennis L. Huff

WU-535-03-10

7. Performing oRganization NaME(S) AND ADDRESS(ES)

National Aeronautics and Space Administration

Lewis Research Center

Cleveland, Ohio 44135-3191
8. Performing organization REPORT NUMBER

E-6811

9. SPONSORING/MONITORING AGENCY NAMES(S) AND ADDRESS(ES)

10. SPONSORING/MONITOAING AGENCY REPORT NUMBER

National Aeronautics and Space Administration

Washington, D.C. 20546-0001

NASA TM- 105406

AIAA-92-0145

11. SUPPLEMENTARY NOTES

Prepared for the 30th Aerospace Sciences Meeting and Exhibit sponsored by the American Institute of Aeronautics and Astronautics, Reno, Nevada, January 6-9, 1992. Responsible person, Dennis L. Huff, (216) 433-3913.

12a. DISTRIBUTION/AVAILABILITY STATEMENT

Unclassified - Unlimited

Subject Category 02

\section{ABSTRACT (Maximum 200 words)}

The unsteady flow field around an oscillating cascade of flat plates is investigated using a time-marching Euler code. Exact solutions based on linear theory serve as model problems to study pressure wave propagation in the numerical solution. The importance of using proper unsteady boundary conditions, grid resolution and time step size is demonstrated. Results show that an approximate non-reflecting boundary condition based on linear theory does a good job of minimizing reflections from the inflow and outflow boundaries and allows the placement of the boundaries to be closer than cases using reflective boundary conditions. Stretching the boundary to dampen the unsteady waves is another way to minimize reflections. Grid clustering near the plates does a better job of capturing the unsteady flow field than cases using uniform grids as long as the CFL number is less than one for a sufficient portion of the grid. Results for various stagger angles and oscillation frequencies show good overall agreement with linear theory as long as the grid is properly resolved.

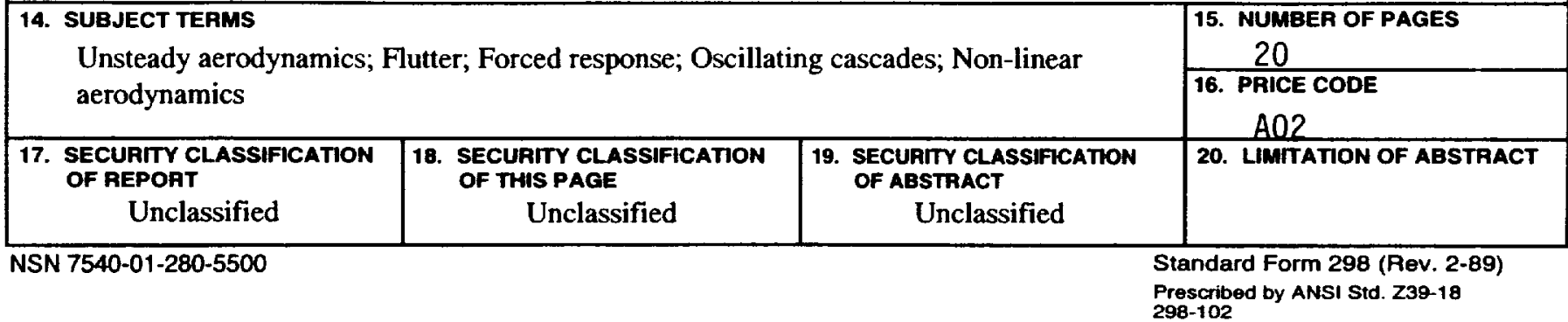

